\title{
TRACE OF INTEGER POWER OF REAL 3 X 3 SPECIFIC MATRICES
}

\author{
Rahmawati $^{1}$, Wartono ${ }^{2}$ and Marhama Jelita ${ }^{3}$ \\ ${ }^{1-2}$ Department of Mathematics \\ ${ }^{3}$ Department of Electrical Engineering \\ Faculty of Sciences and Technology \\ State Islamic University of Sultan Syarif Kasim Riau, Pekanbaru \\ Indonesia
}

\begin{abstract}
Trace matrix is the sum of the main diagonal elements of a square matrix. This study discusses the trace of a positive and negative integer power of a real $3 \times 3$ specific matrix as given in (2). In order to get the trace of negative integer power, the matrix must have an inverse. Determinant and inverse of the matrix are required to generate general formula of the trace negative integer power. Result in this paper, we obtain the general formula of the specific matrix trace with dimension $3 \times 3$ in positive integer power denoted by $\operatorname{Tr}\left(A_{3}{ }^{n}\right)$ or in negative integer power denoted by $\operatorname{Tr}\left(A_{3}{ }^{-n}\right)$. The general formula of $\operatorname{Tr}\left(A_{3}{ }^{n}\right)$ and $\operatorname{Tr}\left(A_{3}{ }^{-n}\right)$ separated into two, for odd and even integers $n$.
\end{abstract}

Key Words: Determinant, Inverse, Multiplication of matrix, Power of matrix, Trace.

\section{INTRODUCTION}

Matrix is the arrangement of numbers in rectangular form. These numbers are called as entries of the matrix. Matrices are categorized into several types including square matrix, identity matrix, diagonal matrix, triangular matrix, symmetrical matrix, and so for. According the matrix theory, there are various types of matrix operations including addition, multiplication, determinant, inverse, trace and so on [1].

In [4], trace of a $n \times n$ matrix $A=\left[a_{i j}\right] ; i, j=1,2, \ldots, n$, is defined to be the sum of the elements on the main diagonal i.e

$$
\operatorname{Tr}(A)=a_{11}+a_{22}+\ldots+a_{n n}
$$

Traces of powers of matrices arise in several fields of mathematics such as matrix theory and numerical algebra. For example, in order to obtain approximations of the smallest and the largest eigenvalues of a symmetric matrix, a procedure based on estimates of the trace $A^{n}$ and $A^{-n}$ for all integers $n$, is proposed in [5]. In the number of theory and combinatoric, traces of powers of integer matrices are connected with the Euler congruence [6],

$$
\operatorname{Tr}\left(M^{p^{r}}\right)=\operatorname{Tr}\left(M^{p^{r-1}}\right) \bmod p^{r}
$$

Holds for all integer matrices $M$, all primes $p$, and all natural $r$.

In its application, traces of matrices are often used in several fields of mathematics, specifically Network Analysis, Number Theory, Dynamic Systems, Matrix Theory, and Differential Problems [2]. In [3] explained that trace of positive integer power of a real $2 \times 2$ matrices, the result being an equation of the general trace form of the matrices. Furthermore, in [4] also discussed the trace of a positive integer power of adjacency matrix. Now we give new theorems to compute trace of specific matrix. In this 
paper, suppose that $A$ is a real $3 \times 3$ specific matrix denoted by $A_{3}$. Our estimation for the trace of $A_{3}{ }^{n}$ and $A_{3}{ }^{-n}$ is based on the multiplication of matrix. Trace of $A_{3}{ }^{-n}$ can be solved if $A_{3}$ invertible, to find $A_{3}{ }^{-1}$ used formula

$$
A_{3}^{-1}=\frac{1}{\operatorname{det}(A)} \operatorname{Adj}(A)
$$

Furthemore, we find the cofactor matrix of $A_{3}$ and adjoin matrix of $A_{3}$ by transposing the cofactor matrix of $A_{3}$. Discussion of determinant matrix, cofactor and adjoin matrix is discussed by [1].

\section{MAIN RESULT}

Suppose that $A$ is a real $3 \times 3$ matrix denoted by $A_{3}$. In this paper, we discuss trace of integer power $A_{3}$, which is trace of positive integer power denoted by $\operatorname{Tr}\left(A_{3}{ }^{n}\right)$ and trace of negative integer power denoted by $\operatorname{Tr}\left(A_{3}{ }^{-n}\right)$ with all integers $n$. This is explained in the following new theorems.

\subsection{Trace of Positive Integer Power of Real Matrices $\boldsymbol{A}_{3}{ }^{n}$}

Theorem 2.1 Let $A_{3}$ is a real $3 \times 3$ matrix as follows

$$
A_{3}=\left[\begin{array}{lll}
0 & a & 0 \\
b & 0 & a \\
0 & 0 & a
\end{array}\right], \forall a, b \in R
$$

Then trace of positive integer power of $A_{3}$ i.e

$$
\operatorname{Tr}\left(A_{3}^{n}\right)=\left\{\begin{array}{cl}
a^{n} & \text {, for odd positive integer } n \\
2(a b)^{\frac{n}{2}}+a^{n} & , \text { for even positive integer } n
\end{array}\right.
$$

Proof. Let $A_{3}$ is a real $3 \times 3$ matrix as in (2), so

$$
\operatorname{Tr}\left(A_{3}\right)=a
$$

Furthermore, we find positive integer power of matrix $\left(A_{3}\right)^{2}$ until $\left(A_{3}\right)^{8}$ and trace each of them

For $n=2,\left(A_{3}\right)^{2}=A_{3} A_{3}=\left[\begin{array}{lll}0 & a & 0 \\ b & 0 & a \\ 0 & 0 & a\end{array}\right]\left[\begin{array}{ccc}0 & a & 0 \\ b & 0 & a \\ 0 & 0 & a\end{array}\right]=\left[\begin{array}{ccc}a b & 0 & a^{2} \\ 0 & a b & a^{2} \\ 0 & 0 & a^{2}\end{array}\right]$

$\operatorname{Tr}\left(A_{3}^{2}\right)=2 a b+a^{2}$

For $n=3,\left(A_{3}\right)^{3}=\left(A_{3}\right)^{2} A_{3}=\left[\begin{array}{ccc}a b & 0 & a^{2} \\ 0 & a b & a^{2} \\ 0 & 0 & a^{2}\end{array}\right]\left[\begin{array}{ccc}0 & a & 0 \\ b & 0 & a \\ 0 & 0 & a\end{array}\right]=\left[\begin{array}{ccc}0 & a^{2} b & a^{3} \\ a b^{2} & 0 & a^{2} b+a^{3} \\ 0 & 0 & a^{3}\end{array}\right]$

$$
\operatorname{Tr}\left(A_{3}^{3}\right)=a^{3}
$$

For $n=4,\left(A_{3}\right)^{4}=\left(A_{3}\right)^{3} A_{3}=\left[\begin{array}{ccc}0 & a^{2} b & a^{3} \\ a b^{2} & 0 & a^{2} b+a^{3} \\ 0 & 0 & a^{3}\end{array}\right]\left[\begin{array}{ccc}0 & a & 0 \\ b & 0 & a \\ 0 & 0 & a\end{array}\right]=\left[\begin{array}{ccc}a^{2} b^{2} & 0 & a^{3} b+a^{4} \\ 0 & a^{2} b^{2} & a^{3} b+a^{4} \\ 0 & 0 & a^{4}\end{array}\right]$ 
International Journal of Advances in Scientific Research and Engineering (ijasre), Vol 5 (3), March-2019

$$
\operatorname{Tr}\left(A_{3}^{4}\right)=2 a^{2} b^{2}+a^{4} .
$$

For $n=5,\left(A_{3}\right)^{5}=\left(A_{3}\right)^{4} A_{3}=\left[\begin{array}{ccc}a^{2} b^{2} & 0 & a^{3} b+a^{4} \\ 0 & a^{2} b^{2} & a^{3} b+a^{4} \\ 0 & 0 & a^{4}\end{array}\right]\left[\begin{array}{lll}0 & a & 0 \\ b & 0 & a \\ 0 & 0 & a\end{array}\right]=\left[\begin{array}{ccc}0 & a^{3} b^{2} & a^{4} b+a^{5} \\ a^{2} b^{3} & 0 & a^{3} b^{2}+a^{4} b+a^{5} \\ 0 & 0 & a^{5}\end{array}\right]$

$$
\operatorname{Tr}\left(A_{3}^{5}\right)=a^{5} .
$$

For $n=6, \quad\left(A_{3}^{6}\right)=\left(A_{3}\right)^{5} A_{3}$

$$
\begin{aligned}
& =\left[\begin{array}{ccc}
0 & a^{3} b^{2} & a^{4} b+a^{5} \\
a^{2} b^{3} & 0 & a^{3} b^{2}+a^{4} b+a^{5} \\
0 & 0 & a^{5}
\end{array}\right]\left[\begin{array}{lll}
0 & a & 0 \\
b & 0 & a \\
0 & 0 & a
\end{array}\right] \\
& =\left[\begin{array}{ccc}
a^{3} b^{3} & 0 & a^{4} b^{2}+a^{5} b+a^{6} \\
0 & a^{3} b^{3} & a^{4} b^{2}+a^{5} b+a^{6} \\
0 & 0 & a^{6}
\end{array}\right] \\
\operatorname{Tr}\left(A_{3}{ }^{6}\right) & =2 a^{3} b^{3}+a^{6} .
\end{aligned}
$$

For $n=7,\left(A_{3}\right)^{7}=\left(A_{3}\right)^{6} A_{3}$

$$
\begin{aligned}
& =\left[\begin{array}{ccc}
a^{3} b^{3} & 0 & a^{4} b^{2}+a^{5} b+a^{6} \\
0 & a^{3} b^{3} & a^{4} b^{2}+a^{5} b+a^{6} \\
0 & 0 & a^{6}
\end{array}\right]\left[\begin{array}{lll}
0 & a & 0 \\
b & 0 & a \\
0 & 0 & a
\end{array}\right] \\
& =\left[\begin{array}{ccc}
0 & a^{4} b^{3} & a^{5} b^{2}+a^{6} b+a^{7} \\
a^{3} b^{4} & 0 & a^{4} b^{3}+a^{5} b^{2}+a^{6} b+a^{7} \\
0 & 0 & a^{7}
\end{array}\right] \\
\operatorname{Tr}\left(A_{3}{ }^{7}\right) & =a^{7} .
\end{aligned}
$$

For $n=8$,

$$
\begin{aligned}
\left(A_{3}\right)^{8} & =\left(A_{3}\right)^{7} A_{3} \\
& =\left[\begin{array}{ccc}
0 & a^{4} b^{3} & a^{5} b^{2}+a^{6} b+a^{7} \\
a^{3} b^{4} & 0 & a^{4} b^{3}+a^{5} b^{2}+a^{6} b+a^{7} \\
0 & 0 & a^{7}
\end{array}\right]\left[\begin{array}{lll}
0 & a & 0 \\
b & 0 & a \\
0 & 0 & a
\end{array}\right] \\
& =\left[\begin{array}{ccc}
a^{4} b^{4} & 0 & a^{5} b^{3}+a^{6} b^{2}+a^{7} b+a^{8} \\
0 & a^{4} b^{4} & a^{5} b^{3}+a^{6} b^{2}+a^{7} b+a^{8} \\
0 & 0 & a^{8}
\end{array}\right] \\
\operatorname{Tr}\left(A_{3}^{8}\right) & =2 a^{4} b^{4}+a^{8} .
\end{aligned}
$$

Based on (3), (5), (7), and (9) we get 
International Journal of Advances in Scientific Research and Engineering (ijasre), Vol 5 (3), March-2019

$$
\begin{aligned}
& \operatorname{Tr}\left(A_{3}\right)=a \\
& \operatorname{Tr}\left(A_{3}{ }^{3}\right)=a^{3} \\
& \operatorname{Tr}\left(A_{3}{ }^{5}\right)=a^{5} \\
& \operatorname{Tr}\left(A_{3}{ }^{7}\right)=a^{7}
\end{aligned}
$$

Such that by observing at the recursive pattren, we find trace of positive integer power of $A_{3}{ }^{n}$ for odd positive integer $n$ i.e

$$
\operatorname{Tr}\left(A_{3}{ }^{n}\right)=a^{n} \text {. }
$$

In the other side, based on (4), (6), (8), and (10), we have

$$
\begin{aligned}
& \operatorname{Tr}\left({A_{3}}^{2}\right)=2 a b+a^{2}=2 a^{\frac{2}{2}} b^{\frac{2}{2}}+a^{2} \\
& \operatorname{Tr}\left(A_{3}^{4}\right)=2 a^{2} b^{2}+a^{4}=2 a^{\frac{4}{2}} b^{\frac{4}{2}}+a^{4} \\
& \operatorname{Tr}\left(A_{3}^{6}\right)=2 a^{3} b^{3}+a^{6}=2 a^{\frac{6}{2}} b^{\frac{6}{2}}+a^{6} \\
& \operatorname{Tr}\left(A_{3}^{8}\right)=2 a^{4} b^{4}+a^{8}=2 a^{\frac{8}{2}} b^{\frac{8}{2}}+a^{8}
\end{aligned}
$$

Such that by observing at the recursive pattren, we find trace of positive integer power of $A_{3}{ }^{n}$ for even positive integer $n$ i.e

$$
\operatorname{Tr}\left(A_{3}{ }^{n}\right)=2 a^{\frac{n}{2}} b^{\frac{n}{2}}+a^{n}=2(a b)^{\frac{n}{2}}+a^{n} .
$$

More generally, based on (11) and (12), general forms $\operatorname{Tr}\left(A_{3}{ }^{n}\right)$ i.e

$$
\operatorname{Tr}\left(A_{3}{ }^{n}\right)=\left\{\begin{array}{cl}
a^{n} & , \text { for odd positive integer } n \\
2(a b)^{\frac{n}{2}}+a^{n} & , \text { for even positive integer } n
\end{array}\right.
$$

\subsection{Trace of Negative Integer Power of Real Matrices $A_{3}^{-n}$}

Theorem 2.2 Suppose that $A_{3}$ is a real $3 \times 3$ matrix as in (2) where $a, b \neq 0$. If $A_{3}$ invertible, then trace of negative integer power of $A_{3}$ i.e

$$
\operatorname{Tr}\left(A_{3}{ }^{-n}\right)=\left\{\begin{array}{cl}
a^{-n} & , \text { for odd positive integer } n \\
a^{-\frac{n}{2}}\left(2^{-\frac{n}{2}}+a^{-\frac{n}{2}}\right) & , \text { for even positive integer } n
\end{array}\right.
$$

Proof. In this section, the initial step to find trace of negative integer power of $A_{3}{ }^{-n}$, we must find determinants of $A_{3}$ in (2) i.e $\operatorname{det}\left(A_{3}\right)=-a^{2} b \neq 0$ and the cofactor matrix of $A_{3}$ is

$$
C=\left[\begin{array}{ccc}
0 & -a b & 0 \\
-a^{2} & 0 & 0 \\
a^{2} & 0 & -a b
\end{array}\right]
$$


Adjoin of matrix $A_{3}$ is $\operatorname{adj}\left(A_{3}\right)=C^{T}$

$$
\operatorname{adj}\left(A_{3}\right)=\left[\begin{array}{ccc}
0 & -a^{2} & a^{2} \\
-a b & 0 & 0 \\
0 & 0 & -a b
\end{array}\right] .
$$

Then,

$$
\begin{aligned}
& \left(A_{3}\right)^{-1}=\frac{1}{\operatorname{det}\left(A_{3}\right)} \operatorname{adj}\left(A_{3}\right)=\frac{1}{-a^{2} b}\left[\begin{array}{ccc}
0 & -a^{2} & a^{2} \\
-a b & 0 & 0 \\
0 & 0 & -a b
\end{array}\right]=\left[\begin{array}{ccc}
0 & \frac{1}{b} & -\frac{1}{b} \\
\frac{1}{a} & 0 & 0 \\
0 & 0 & \frac{1}{a}
\end{array}\right] \\
& \operatorname{Tr}\left(A_{3}{ }^{-1}\right)=\frac{1}{a}
\end{aligned}
$$

Furthermore, we find negative integer power of matrix $\left(A_{3}\right)^{-2}$ until $\left(A_{3}\right)^{-8}$ and trace each of them

$$
\begin{aligned}
& \text { For } n=2,\left(A_{3}\right)^{-2}=\left(A_{3}\right)^{-1}\left(A_{3}\right)^{-1}=\left[\begin{array}{ccc}
0 & \frac{1}{b} & -\frac{1}{b} \\
\frac{1}{a} & 0 & 0 \\
0 & 0 & \frac{1}{a}
\end{array}\right]\left[\begin{array}{ccc}
0 & \frac{1}{b} & -\frac{1}{b} \\
\frac{1}{a} & 0 & 0 \\
0 & 0 & \frac{1}{a}
\end{array}\right]=\left[\begin{array}{ccc}
\frac{1}{a b} & 0 & -\frac{1}{a b} \\
0 & \frac{1}{a b} & -\frac{1}{a b} \\
0 & 0 & \frac{1}{a^{2}}
\end{array}\right] \\
& \qquad \operatorname{Tr}\left(A_{3}^{-2}\right)=\frac{2}{a b}+\frac{1}{a^{2}} \text {. }
\end{aligned}
$$

For $n=3,\left(A_{3}\right)^{-3}=\left(A_{3}\right)^{-2}\left(A_{3}\right)^{-1}=\left[\begin{array}{ccc}\frac{1}{a b} & 0 & -\frac{1}{a b} \\ 0 & \frac{1}{a b} & -\frac{1}{a b} \\ 0 & 0 & \frac{1}{a^{2}}\end{array}\right]\left[\begin{array}{ccc}0 & \frac{1}{b} & -\frac{1}{b} \\ \frac{1}{a} & 0 & 0 \\ 0 & 0 & \frac{1}{a}\end{array}\right]=\left[\begin{array}{ccc}0 & \frac{1}{a b^{2}} & -\frac{1}{a b^{2}}-\frac{1}{a^{2} b} \\ \frac{1}{a^{2} b} & 0 & -\frac{1}{a^{2} b} \\ 0 & 0 & \frac{1}{a^{3}}\end{array}\right]$

$$
\operatorname{Tr}\left(A_{3}{ }^{-3}\right)=\frac{1}{a^{3}}
$$

For $n=4$, 
International Journal of Advances in Scientific Research and Engineering (ijasre), Vol 5 (3), March-2019

$$
\begin{aligned}
\left(A_{3}\right)^{-4} & =\left(A_{3}\right)^{-3}\left(A_{3}\right)^{-1} \\
& =\left[\begin{array}{ccc}
0 & \frac{1}{a b^{2}} & -\frac{1}{a b^{2}}-\frac{1}{a^{2} b} \\
\frac{1}{a^{2} b} & 0 & -\frac{1}{a^{2} b} \\
0 & 0 & \frac{1}{a^{3}}
\end{array}\right]\left[\begin{array}{ccc}
0 & \frac{1}{b} & -\frac{1}{b} \\
\frac{1}{a} & 0 & 0 \\
0 & 0 & \frac{1}{a}
\end{array}\right] \\
& =\left[\begin{array}{ccc}
\frac{1}{a^{2} b^{2}} & 0 & -\frac{1}{a^{2} b^{2}}-\frac{1}{a^{3} b} \\
0 & \frac{1}{a^{2} b^{2}} & -\frac{1}{a^{2} b^{2}}-\frac{1}{a^{3} b} \\
0 & 0 & \frac{1}{a^{4}}
\end{array}\right] \\
\operatorname{Tr}\left(A_{3}{ }^{-4}\right) & =\frac{2}{a^{2} b^{2}}+\frac{1}{a^{4}}
\end{aligned}
$$

For $n=5$,

$$
\begin{aligned}
& \left(A_{3}\right)^{-5}=\left(A_{3}\right)^{-4}\left(A_{3}\right)^{-1} \\
& =\left[\begin{array}{ccc}
\frac{1}{a^{2} b^{2}} & 0 & -\frac{1}{a^{2} b^{2}}-\frac{1}{a^{3} b} \\
0 & \frac{1}{a^{2} b^{2}} & -\frac{1}{a^{2} b^{2}}-\frac{1}{a^{3} b} \\
0 & 0 & \frac{1}{a^{4}}
\end{array}\right]\left[\begin{array}{ccc}
0 & \frac{1}{b} & -\frac{1}{b} \\
\frac{1}{a} & 0 & 0 \\
0 & 0 & \frac{1}{a}
\end{array}\right] \\
& =\left[\begin{array}{ccc}
0 & \frac{1}{a^{2} b^{3}} & -\frac{1}{a^{2} b^{3}}-\frac{1}{a^{3} b^{2}}-\frac{1}{a^{4} b} \\
\frac{1}{a^{3} b^{2}} & 0 & -\frac{1}{a^{3} b^{2}}-\frac{1}{a^{4} b} \\
0 & 0 & \frac{1}{a^{5}}
\end{array}\right] \\
& \operatorname{Tr}\left(A_{3}{ }^{-5}\right)=\frac{1}{a^{5}}
\end{aligned}
$$

For $n=6$,

$$
\begin{aligned}
\left(A_{3}\right)^{-6} & =\left(A_{3}\right)^{-5}\left(A_{3}\right)^{-1} \\
& =\left[\begin{array}{ccc}
0 & \frac{1}{a^{2} b^{3}} & -\frac{1}{a^{2} b^{3}}-\frac{1}{a^{3} b^{2}}-\frac{1}{a^{4} b} \\
\frac{1}{a^{3} b^{2}} & 0 & -\frac{1}{a^{3} b^{2}}-\frac{1}{a^{4} b} \\
0 & 0 & \frac{1}{a^{5}}
\end{array}\right]\left[\begin{array}{ccc}
0 & \frac{1}{b} & -\frac{1}{b} \\
\frac{1}{a} & 0 & 0 \\
0 & 0 & \frac{1}{a}
\end{array}\right] \\
& =\left[\begin{array}{ccc}
\frac{1}{a^{3} b^{3}} & 0 & -\frac{1}{a^{3} b^{3}}-\frac{1}{a^{4} b^{2}}-\frac{1}{a^{5} b} \\
0 & \frac{1}{a^{3} b^{3}} & -\frac{1}{a^{3} b^{3}}-\frac{1}{a^{4} b^{2}}-\frac{1}{a^{5} b} \\
0 & 0 & \frac{1}{a^{6}}
\end{array}\right]
\end{aligned}
$$


International Journal of Advances in Scientific Research and Engineering (ijasre), Vol 5 (3), March-2019

$$
\operatorname{Tr}\left(A_{3}{ }^{-6}\right)=\frac{2}{a^{3} b^{3}}+\frac{1}{a^{6}}
$$

For $n=7$,

$$
\begin{aligned}
& \left(A_{3}\right)^{-7}=\left(A_{3}\right)^{-6}\left(A_{3}\right)^{-1} \\
& =\left[\begin{array}{ccc}
\frac{1}{a^{3} b^{3}} & 0 & -\frac{1}{a^{3} b^{3}}-\frac{1}{a^{4} b^{2}}-\frac{1}{a^{5} b} \\
0 & \frac{1}{a^{3} b^{3}} & -\frac{1}{a^{3} b^{3}}-\frac{1}{a^{4} b^{2}}-\frac{1}{a^{5} b} \\
0 & 0 & \frac{1}{a^{6}}
\end{array}\right]\left[\begin{array}{ccc}
0 & \frac{1}{b} & -\frac{1}{b} \\
\frac{1}{a} & 0 & 0 \\
0 & 0 & \frac{1}{a}
\end{array}\right] \\
& =\left[\begin{array}{ccc}
0 & \frac{1}{a^{3} b^{4}} & -\frac{1}{a^{3} b^{4}}-\frac{1}{a^{4} b^{3}}-\frac{1}{a^{5} b^{2}}-\frac{1}{a^{6} b} \\
\frac{1}{a^{4} b^{3}} & 0 & -\frac{1}{a^{4} b^{3}}-\frac{1}{a^{5} b^{2}}-\frac{1}{a^{6} b} \\
0 & 0 & \frac{1}{a^{7}}
\end{array}\right] \\
& \operatorname{Tr}\left(A_{3}{ }^{-7}\right)=\frac{1}{a^{7}}
\end{aligned}
$$

For $n=8$,

$$
\begin{aligned}
& \left(A_{3}\right)^{-8}=\left(A_{3}\right)^{-7}\left(A_{3}\right)^{-1} \\
& =\left[\begin{array}{ccc}
0 & \frac{1}{a^{3} b^{4}} & -\frac{1}{a^{3} b^{4}}-\frac{1}{a^{4} b^{3}}-\frac{1}{a^{5} b^{2}}-\frac{1}{a^{6} b} \\
\frac{1}{a^{4} b^{3}} & 0 & -\frac{1}{a^{4} b^{3}}-\frac{1}{a^{5} b^{2}}-\frac{1}{a^{6} b} \\
0 & 0 & \frac{1}{a^{7}}
\end{array}\right]\left[\begin{array}{ccc}
0 & \frac{1}{b} & -\frac{1}{b} \\
\frac{1}{a} & 0 & 0 \\
0 & 0 & \frac{1}{a}
\end{array}\right] \\
& =\left[\begin{array}{ccc}
\frac{1}{a^{4} b^{4}} & 0 & -\frac{1}{a^{4} b^{4}}-\frac{1}{a^{5} b^{3}}-\frac{1}{a^{6} b^{2}}-\frac{1}{a^{7} b} \\
0 & \frac{1}{a^{4} b^{4}} & -\frac{1}{a^{4} b^{4}}-\frac{1}{a^{5} b^{3}}-\frac{1}{a^{6} b^{2}}-\frac{1}{a^{7} b} \\
0 & 0 & \frac{1}{a^{8}}
\end{array}\right] \\
& \operatorname{Tr}\left(A_{3}^{-8}\right)=\frac{2}{a^{4} b^{4}}+\frac{1}{a^{8}}
\end{aligned}
$$

Based on (13), (15), (17), and (19) we get

$$
\begin{aligned}
& \operatorname{Tr}\left(A_{3}^{-1}\right)=\frac{1}{a} \\
& \operatorname{Tr}\left(A_{3}^{-3}\right)=\frac{1}{a^{3}} \\
& \operatorname{Tr}\left(A_{3}^{-5}\right)=\frac{1}{a^{5}}
\end{aligned}
$$


International Journal of Advances in Scientific Research and Engineering (ijasre), Vol 5 (3), March-2019

$$
\operatorname{Tr}\left(A_{3}^{-7}\right)=\frac{1}{a^{7}}
$$

Such that by observing at the recursive pattren, we find trace of negative integer power of $A_{3}^{-n}$ for odd number $n$ i.e

$$
\operatorname{Tr}\left(A_{3}^{-n}\right)=\frac{1}{a^{n}}=a^{-n}
$$

In the other side, based on (14), (16), (18), and (20) we obtain

$$
\begin{aligned}
& \operatorname{Tr}\left(A_{3}{ }^{-2}\right)=\frac{2}{a b}+\frac{1}{a^{2}}=\frac{2}{a^{\frac{2}{2}} b^{\frac{2}{2}}}+\frac{1}{a^{2}} \\
& \operatorname{Tr}\left(A_{3}{ }^{-4}\right)=\frac{2}{a^{2} b^{2}}+\frac{1}{a^{4}}=\frac{2}{a^{\frac{4}{2}} b^{\frac{4}{2}}}+\frac{1}{a^{4}} \\
& \operatorname{Tr}\left(A_{3}{ }^{-6}\right)=\frac{2}{a^{3} b^{3}}+\frac{1}{a^{6}}=\frac{2}{\frac{6}{a^{2}} b^{\frac{6}{2}}}+\frac{1}{a^{6}} \\
& \operatorname{Tr}\left(A_{3}{ }^{-8}\right)=\frac{2}{a^{4} b^{4}}+\frac{1}{a^{8}}=\frac{2}{a^{\frac{8}{2}} b^{\frac{8}{2}}}+\frac{1}{a^{8}}
\end{aligned}
$$

Such that by observing at the recursive pattren, we find trace of negative integer power of $A_{3}^{-n}$ for even number $n$ i.e

$$
\operatorname{Tr}\left(A_{3}{ }^{n}\right)=\frac{2}{a^{\frac{n}{2}} b^{\frac{n}{2}}}+\frac{1}{a^{n}}=a^{-\frac{n}{2}}\left(2 b^{-\frac{n}{2}}+a^{-\frac{n}{2}}\right)
$$

More generally, based on (21) and (22), general forms $\operatorname{Tr}\left({A_{3}}^{-n}\right)$ i.e

$$
\operatorname{Tr}\left(A_{3}{ }^{-n}\right)=\left\{\begin{array}{cl}
a^{-n} & , \text { for odd positive integer } n \\
a^{-\frac{n}{2}}\left(2 b^{-\frac{n}{2}}+a^{-\frac{n}{2}}\right) & \text {, for even positive integer } n
\end{array}\right.
$$

Example. Suppose that $A_{3}=\left[\begin{array}{lll}0 & 1 & 0 \\ 2 & 0 & 1 \\ 0 & 0 & 1\end{array}\right]$ is an invertible, let we are to find $\operatorname{Tr}\left(A^{100}\right)$ and $\operatorname{Tr}\left(A^{-2019}\right)$.

In this case, $a=1, b=2$ and for $n=100$, which is even, hence by Theorem 2.1, we get $\operatorname{Tr}\left(A_{3}^{100}\right)=2(1 \times 2)^{50}=2^{51}$.

In the other side, because $A_{3}$ invertible, then by Theorem 2.2, for odd integer $n=2019$, we get $\operatorname{Tr}\left(A_{3}{ }^{-2019}\right)=1^{-2019}=1$.

\section{CONCLUSION AND FUTURE WORK}

Based on the results and discussion section, we obtained the general form of Trace $A_{3}$ as given in (2) for negative and positive integers power of $n$. In the future, we suggest that the readers continue the study for matrix with sizes larger than $3 \times 3$ or the trace of integer power matrix with matrix form something else. 


\section{REFERENCES}

1. H. Anton, "Elementary Linear Algebra", $5^{\text {th }}$ ed, John Willey \& Sons, New York. 1987.

2. C. Brezinski, P. Fika, and M. Mitrouli, "Estimations of the Trace of Powers of Positive Self-Adjoint Operators by Extrapolation of the Moments", Electronic Transactions on Numerical Analysis, vol. 39, 2012, pp. 144-155.

3. J. Pahade, and M. Jha, “ Trace of Positive integer Power of Real 2 x 2 Matrices”, Advances in Linear Algebra \& Matrix Theory, vol. 5, 2015, pp. 150-155.

4. J. K. Pahade, and M. Jha, “ Trace of Positive integer Power of Adjacency Matrices”, Global Journal of Pure and Applied Mathematics, vol. 13,6, 2017, pp. 2079-2087.

5. V. Pan, "Estimating the Extremal Eigenvalues of a Symmetric Matrix," Computers \& Mathematics with Applications, vol. 20, issue 2, 1990, pp. 17-22.

6. A.V. Zarelua, "On Congruences for the Traces of Powers of Some Matrices", Proceedings of the Steklov Institute of Mathematics, vol. 263, 2008, pp.78-98. 